\title{
Upregulation of the GABA transporter GAT- 1 in the gracile nucleus in the spared nerve injury model of neuropathic pain
}

\author{
Romain-Daniel Gosselin ${ }^{\mathrm{a}, \mathrm{b}, *}$, Damien Bebber ${ }^{\mathrm{a}, \mathrm{b}}$, Isabelle Decosterd ${ }^{\mathrm{a}, \mathrm{b}}$ \\ a Pain Research Unit, Department of Anesthesiology, University Hospital Center and University of Lausanne, Bugnon 46, 1011 Lausanne, Switzerland \\ ${ }^{b}$ Department of Cell Biology and Morphology, University of Lausanne, Bugnon 9, 1005 Lausanne, Switzerland
}

\section{A R T I C L E I N F O}

\section{Article history:}

Received 11 May 2010

Received in revised form 3 June 2010

Accepted 4 June 2010

\section{Keywords:}

Neuropathic pain

Glia

Astrocytes

Gracile

GABA

Transporter

\begin{abstract}
A B S T R A C T
Neuropathic pain is a major health issue and is frequently accompanied by allodynia (painful sensations in response to normally non-painful stimulations), and unpleasant paresthesia/dysesthesia, pointing to alterations in sensory pathways normally dedicated to the processing of non-nociceptive information. Interestingly, mounting evidence indicate that central glial cells are key players in allodynia, partly due to changes in the astrocytic capacity to scavenge extracellular glutamate and $\gamma$-aminobutyric acid (GABA), through changes in their respective transporters (EAAT and GAT). In the present study, we investigated the glial changes occurring in the dorsal column nuclei, the major target of normally innocuous sensory information, in the rat spared nerve injury (SNI) model of neuropathic pain. We report that together with a robust microglial and astrocytic reaction in the ipsilateral gracile nucleus, the GABA transporter GAT- 1 is upregulated with no change in GAT-3 or glutamate transporters. Furthermore, $\left[{ }^{3} \mathrm{H}\right]$ GABA reuptake on crude synaptosome preparation shows that transporter activity is functionally increased ipsilaterally in SNI rats. This GAT-1 upregulation appears evenly distributed in the gracile nucleus and colocalizes with astrocytic activation. Neither glial activation nor GAT-1 modulation was detected in the cuneate nucleus. Together, the present results point to GABA transport in the gracile nucleus as a putative therapeutic target against abnormal sensory perceptions related to neuropathic pain.
\end{abstract}

(c) 2010 Elsevier Ireland Ltd. All rights reserved.
Chronic neuropathic pain (arising from damages to the nervous system) is prevalent, highly debilitating as well as frequently intractable and therefore represents a major socio-economic issue worldwide. It is characterized by prominent features including spontaneous pain, allodynia (painful sensation in response to normally innocuous stimuli) and dysesthesia/paresthesia (unpleasant abnormal sense of touch or sensory experiences such as feeling of numbness, tingling/picking). Remarkably, over the past few years, a large body of evidence has accumulated showing that central glial cells (chiefly astrocytes and microglia) are involved in neuropathic pain $[9,12]$. Indeed, many studies have recurrently indicated that peripheral nerve insults result in profound morphological changes in spinal glial cells as well as upregulations of canonical markers such as Glial Fibrilary Acidic Protein (GFAP) in astrocytes or Ionized calcium Binding Adaptor molecule 1 (Iba-1) in microglia. In addition, many lines of pharmacological evidence point to this so-called spinal "glial reaction" as a key driving force in the persistence of allodynia. Yet, despite significant advances in

\footnotetext{
* Corresponding author. Tel.: +41 21692 5269; fax: +41 216925275.

E-mail addresses: Romain-Daniel.Gosselin@chuv.ch, Romain.Gosselin@unil.ch (R.-D. Gosselin)

URL: http://www.unil.ch/fbm/page28983.html (R.-D. Gosselin).
}

our understanding of the molecular cascades occurring in spinal glia that could drive allodynic behaviors, the exact chain of events remains largely incompletely understood. One hypothesis that has arisen lately points to alterations in the capacity of astrocytes to scavenge synaptically released neurotransmitters (glutamate and GABA) [18]. This may occur through changes in related Excitatory Amino-Acid (EAAT) and GABA (GAT) Transporters, especially a decrease in EAAT-1 and EAAT-2 as well as an increase in GAT-1 and 3 subtypes, known to be expressed in glia, thus impairing the maintenance of a proper excitation over inhibition balance in the somatosensory neuronal network.

Interestingly, it is classically considered that the main first synaptic relay of sensory neurons that code for normally innocuous information (light touch, pressure, vibration) is not located in the dorsal spinal cord (as opposed to the nociceptive neurons). Such information is instead conveyed via the dorsal column pathway toward the gracile and cuneate nuclei, located in the medulla oblongata. Strikingly, some scarce evidence has been provided indicating that the gracile nucleus is also involved in the transmission of nociceptive information or may contribute to mechanical allodynia, especially in neuropathic conditions $[10,11,14]$. Furthermore, consistent, albeit rare, reports have highlighted the existence of a microglial and an astrocytic reaction in the gracile nucleus following neuropathy $[6,8,19]$. However, nothing is known regarding the 
expression of glutamate and GABA transporters in this brain region in neuropathic pain.

In the present study, we induced peripheral neuropathy using the spared nerve injury (SNI) model in rats in order to study the glial reaction and the expression of EAAT and GAT transporters in the gracile nucleus. The results indicate that, together with a microglial and astrocytic activation, a significant increase in GAT-1 expression occurs in the entirety of the ipsilateral gracile nucleus, with no detectable change in GAT-3, EAAT- 1 or EAAT-2 expression.

Adult Sprague-Dawley rats, weighing 200-250 g (Charler River, France) were submitted to SNI surgery as previously described [3]. Briefly, under isoflurane anesthesia, the left sciatic nerve was exposed at the level of its trifurcation into sural, common peroneal and tibial branches. The common peroneal and tibial nerves were ligated and cut leaving the sural nerve intact. Wounds were sutured and the animals were left to recover and watched daily for any sign of stress, distress or autotomy. Sham surgery consisted in the same procedure except that all nerves were left untouched before the suturing step. Tissues were collected seven days post surgery under deep lethal pentobarbital injection. All procedures were approved by the Committee on Animal Experimentation for the canton of Vaud, Switzerland, in line with Swiss Federal Law on Animal Welfare and the recommendations of the International Association for the Study of Pain.

For immunofluorescence, rats under terminal anesthesia were perfused with phosphate buffered saline for $1 \mathrm{~min}$ and then with ice-cold paraformaldehyde ( $4 \% \mathrm{w} / \mathrm{v}$ in PBS) for $10 \mathrm{~min}$. The brains were carefully dissected and post fixed in the same fixative overnight at $4{ }^{\circ} \mathrm{C}$. Free floating coronal sections ( $30 \mu \mathrm{m}$ thick) of the medulla oblongata were cut from the caudal to the rostral extremity using a vibratome (Leica), collected in cold PBS, and stored at $4{ }^{\circ} \mathrm{C}$ until use. For mapping experiments, each slice was stored separately starting at the first one were gracile nuclei were visible under a binocular lens (around $-14.6 \mathrm{~mm}$ from Bregma, noted level $0 \mu \mathrm{m}$ ) until the last one (around $-13.7 \mathrm{~mm}$ from Bregma, noted level $990 \mu \mathrm{m}$ ). For immunolabeling, all incubation solutions were prepared from the same solution as for blocking, consisting in PBS supplemented with $10 \%$ normal goat serum and $0.05 \%$ Triton $\mathrm{X}-100$. The same antibodies as for Western-blot were used as follows: EAAT-1 (1/500), EAAT-2 (1/50; Abcam, USA), GAT-1 (1/500; Abcam, USA), GAT-3 (1/500; Abcam, USA), GFAP (1/1000; Millipore, USA). In addition, the following antibodies were used: rabbit antiIba1 (1/200; Wako, Japan), mouse anti-cd11b (1/500; Setotec, UK). Secondary antibodies were Goat anti-rabbit Alexa-488 and Goat anti-mouse Cy3 (1/1000) and were used together with DAPI nuclear labeling. Blocking, primary and secondary incubations were $30 \mathrm{~min}$ at room temperature, over night at $4{ }^{\circ} \mathrm{C}$ and $1 \mathrm{~h}$ at room temperature respectively. Microphotographs were taken using an epifluorescent microscope (AxioPlan, Zeiss, Switzerland) and the Axiovision software (Zeiss). High magnification images $(\times 40$ objective) were taken using $z$-stack option (stacks of 10 images). This allowed the stacking and deconvoluated acquisition of photographs along the whole thickness of the slice. For each section, digital images were all processed using the same settings to improve the contrasts with no separated part of any pictures modified independently. For signal quantification, the images were analyzed by an experimenter blind to both animal group and body side of the sample using ImageJ freeware (National Institute of Health, http://rsb.info.nih.gov/ij/). Mean pixel intensity (grey value) of GFAP or GAT-1 immunoreactivities was calculated by selecting a $0.04 \mathrm{~mm}^{2}$ area in the center portion of the gracile or cuneate nucleus. Statistics were made using GraphPadPrism software (GraphPad, USA). Student's $t$ test was performed to compare sham and SNI samples, significance threshold was set up at $p<0.05$ in all analyses.

For Western-blot analysis, rats under terminal anesthesia were decapitated and the brains were rapidly dissected to expose the medulla oblongata. Isolated samples consisted in roughly $2 \mathrm{~mm}$ long pieces of dorso-medial medullar tissue underneath the caudal limit of the cerebellum. Ipsilateral and contralateral samples were collected separately. Following protein extraction, Western-blots were performed using the following antibodies: rabbit against EAAT-1 (1/5000; Abcam, USA), rabbit against EAAT2 (1/500; Abcam, USA), rabbit against GAT-1 (1/5000; Abcam, USA), rabbit against GAT-3 (1/5000; Abcam, USA), mouse antiGFAP (1/2000; Millipore, USA), rabbit against Iba1 (1/1000; Wako, Japan) and mouse anti-GAPDH (1/50,000; Abcam, USA). Horse radish peroxidase-conjugated secondary antibodies were used for detection (1/8000, Dako, Denmark). Blocking (30 min at room temperature) and probing ( $1 \mathrm{~h}$ at room temperature) incubations were made in $5 \%(\mathrm{w} / \mathrm{v})$ non-fat milk in phosphate buffered saline (PBS) with $0.1 \%$ Tween 20 . Washings were made in PBS-Tween. After washings, the membranes were incubated with ECL reagent (Pierce, USA) and exposed to a luminescent image analyzer (LAS-4000, Fujifilm, Bucher, Switzerland). For subsequent GAPDH detection, the membranes were subsequently directly processed for antibody incubation. For quantification, the bands were quantified using ImageJ software, signals were first normalized over GAPDH intensity and then over the mean of sham intensity. Statistics were made using GraphPadPrism software (GraphPad, USA). Student's $t$ test was used and threshold was set up at $p<0.05$ in all analyses.

For preparation of crude synaptosomes, about $5 \mathrm{~mm}$ of ipsilateral and contralateral brainstem dorsal portion (straddling the caudal extremity of the fourth ventricle) were collected in ice-cold homogenization buffer ( $0.32 \mathrm{M}$ sucrose in $10 \mathrm{mM}$ HEPES). Samples were homogenized by a $10 \mathrm{~s}$ stroke with a rotator Miccra D1 homogenizer (Milian, Switzerland) and centrifuged (1000 g, $10 \mathrm{~min}$ at $4{ }^{\circ} \mathrm{C}$ ) to pellet cell debris and nuclei. Supernatants were then centrifuged $\left(15,000 \mathrm{~g}, 10 \mathrm{~min}\right.$ at $\left.4{ }^{\circ} \mathrm{C}\right)$ and the pellets were resuspended in incubation buffer consisting in (mM): $\mathrm{KCl}: 5.33 ; \mathrm{NaHCO}_{3}: 26.2$; $\mathrm{NaCl}$ : 117.2; $\mathrm{NaH}_{2} \mathrm{PO}_{4}$ : 1 ; D-glucose: $5.5 ; \mathrm{CaCl}_{2}$ : $1.2 ;$ HEPES: $1 ; \mathrm{pH}$ 7.4. Crude synaptosomes were exposed $5 \mathrm{~min}$ (room temperature) to either GAT inhibitor NNC711 (Tocris, $1 \mu \mathrm{M}$ ) or the same volume of incubation buffer (vehicle). Subsequently, all samples were incubated with $\left[{ }^{3} \mathrm{H}\right]$ GABA (PerkinElmer, $50 \mathrm{nM}, 89.5 \mathrm{Ci} / \mathrm{mmol}$ ) for $5 \mathrm{~min}$. Reuptake was stopped by centrifugation $(15,000 \mathrm{~g}, 1 \mathrm{~min}$ at $4^{\circ} \mathrm{C}$ ) followed by 3 cycles washings/resuspensions/centrifugation in ice-cold incubation buffer. Synaptosomes were lysed in $300 \mu \mathrm{l}$ of $\mathrm{NaOH} 0.25 \mathrm{M}$, SDS $0.1 \%$. The incorporated radioactivity was quantified by liquid scintillation and protein content was evaluated by Bradford reaction.

In SNI animals, an augmentation in the density of Iba1 expressing microglia is noticeable ipsilaterally to the injury (Fig. 1A panels a, b). Furthermore, an astrocytic reaction is also evident as assessed by the intensification in GFAP immunoreactivity (Fig. 1A panels $c$, d). Additionally, a rise in cell density is also visible, as shown by the increased nuclei packing observed using DAPI labeling (Fig. 1A panels e, f). This increase in cell density might be attributable to processes of cell proliferation or division, both phenomena being known to occur in the spinal cord following neuropathy. In this regard, paralleling what has been described in the spinal cord, the cell type accountable for this augmentation is likely to be microglia and, to a lesser extent, astrocytes $[4,16]$. When considering single coronal plans, the glial reaction seems to be evenly distributed throughout the gracile parenchyma. No difference in glial markers was detected between the gracile nuclei from sham animals and the contralateral one from SNI rats (not shown). Western-blot analysis confirmed the increase in GFAP in the ipsilateral tissue from neuropathic rats in comparison to sham animals $(p=0.05, n=7$ Student's $t$ test, Fig. 1B). Additionally, the study of GAT- 1 protein showed a significant increase in GAT-1 in the ipsilateral side of SNI animals compared to the sham condition $(p<0.0001$, Student's $t$ test, 
(A)
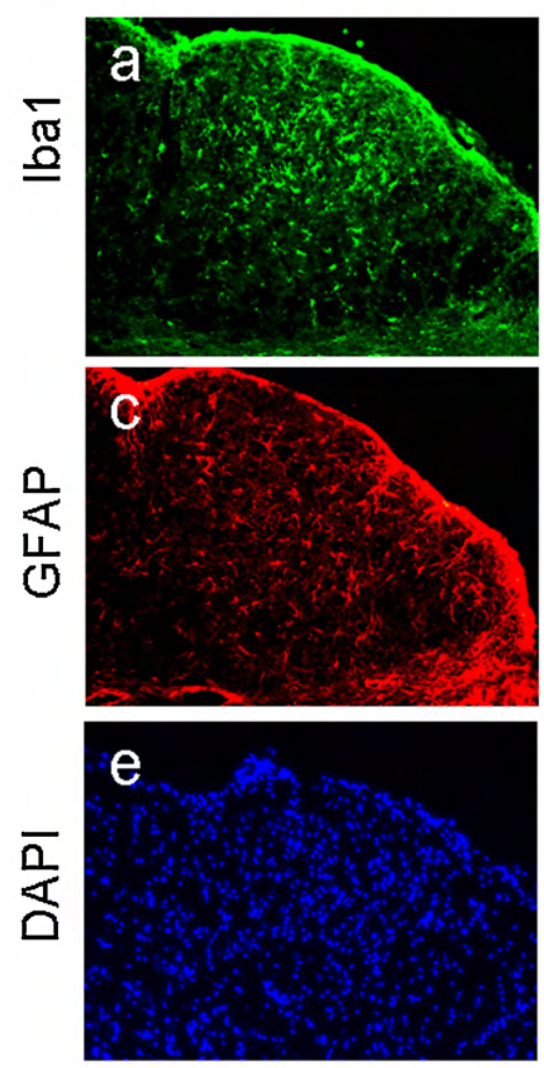

(B)
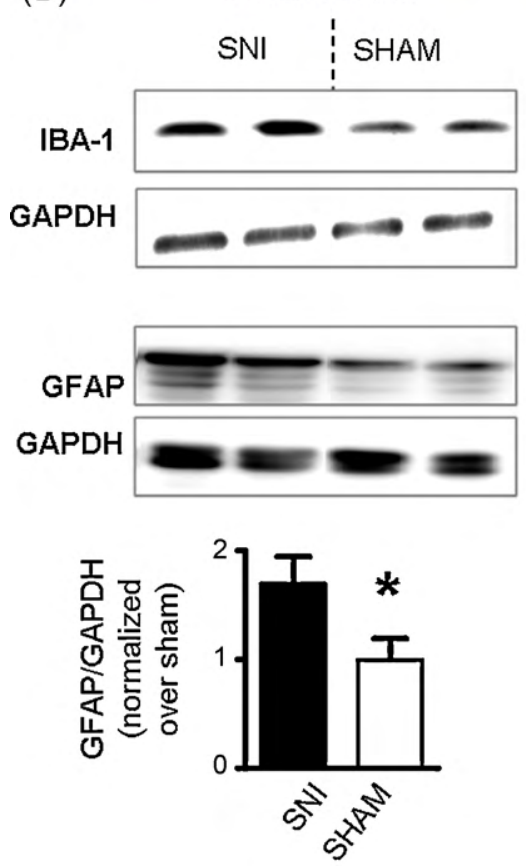

CONTRALATERAL
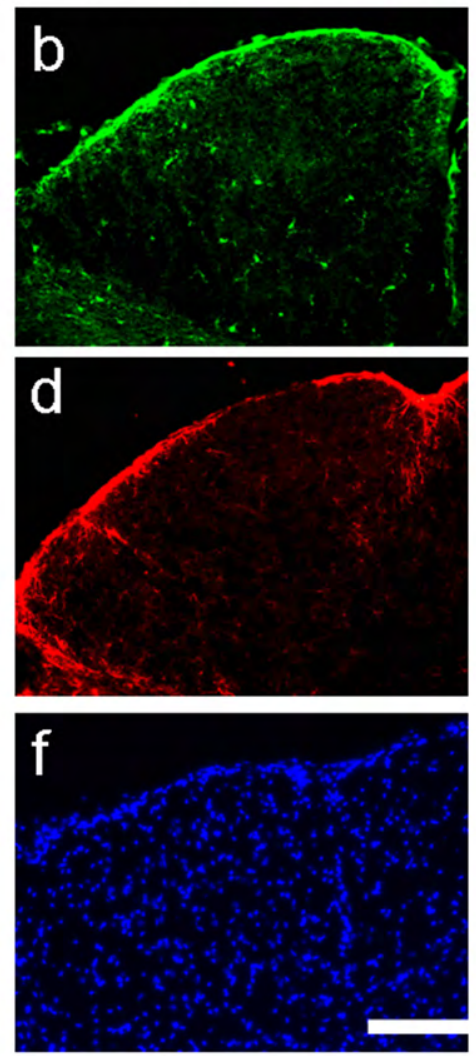

CONTRALATERAL
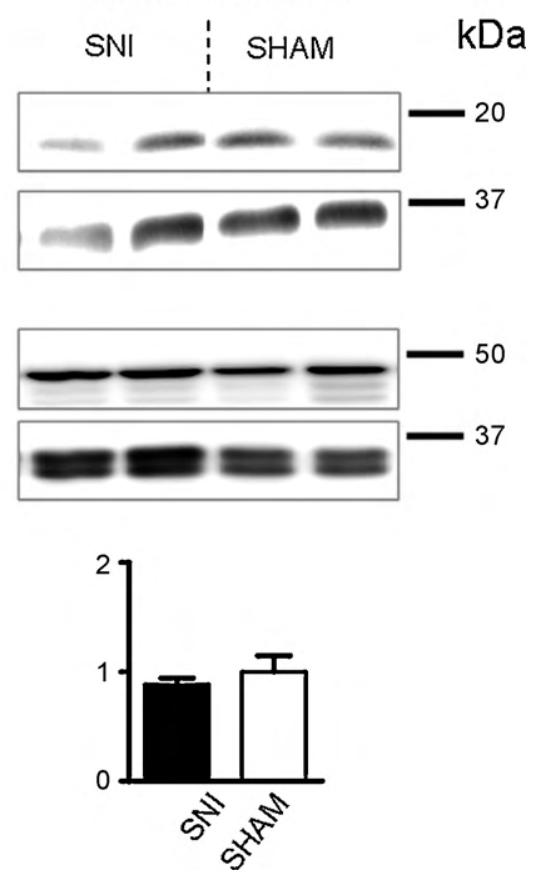

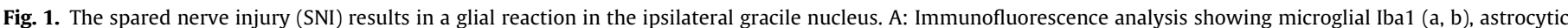

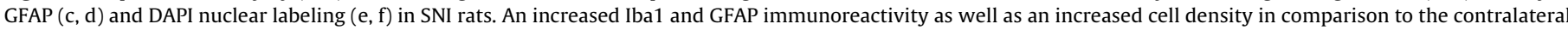

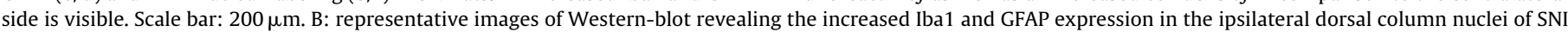
rats. Bar histograms show GFAP band intensity, as a ratio over GAPDH and normalized over sham. ${ }^{*}, p<0.05$ in Student's $t$ test, $n=7$ in each group.

$n=7$ in each group, Fig. 2A, B). No significant difference was found regarding EAAT-1, EAAT-2 or GAT-3 between samples from sham and SNI animals, either ipsilaterally or contralaterally (Fig. 2A). Furthermore, functional experiments on synaptosomes show that in SNI animals, the ipsilateral dorsal brainstem (containing the gracile nucleus) incorporates more $\left[{ }^{3} \mathrm{H}\right] \mathrm{GABA}$ than contralateral tissue $\left(2.49 \times 10^{6} \pm 274,000\right.$ vs. $1.43 \times 10^{6} \pm 327,000 \mathrm{cpm} / \mathrm{mg}$ proteins, $p<0.01$ ANOVA followed by Dunnett's test, $n=6$ in each 
(A)

IPSILATERAL

GAT-1

GAT-3

EAAT-1

EAAT-2

GAPDH

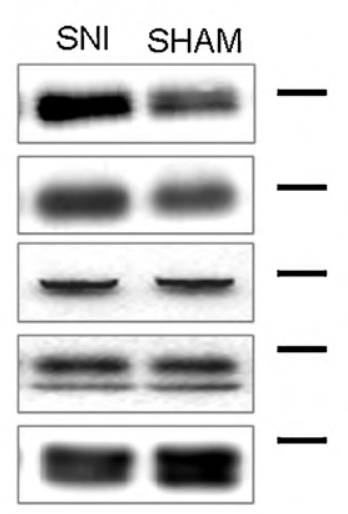

(B)
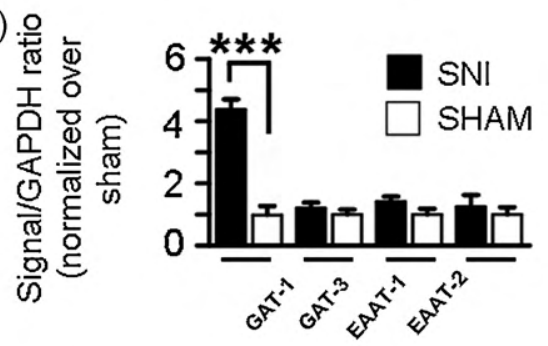

CONTRALATERAL
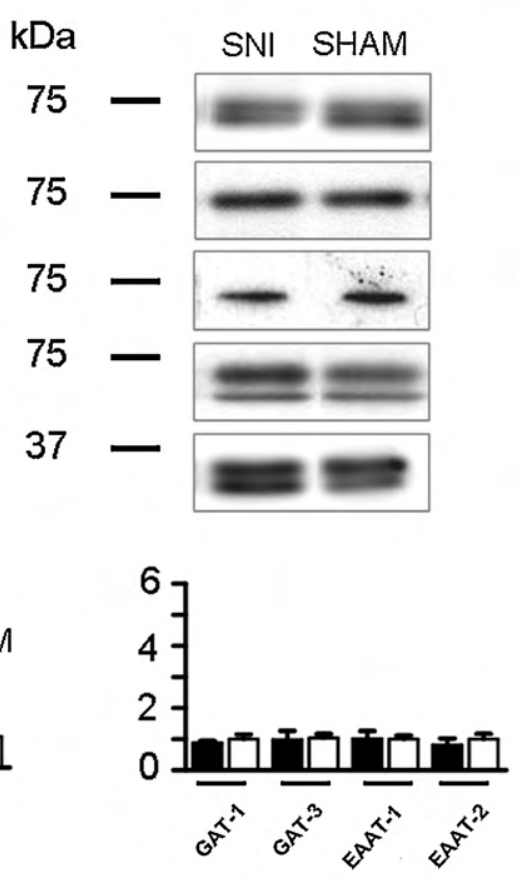

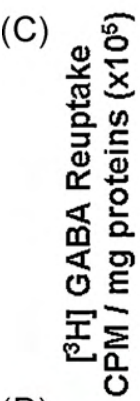

(D)
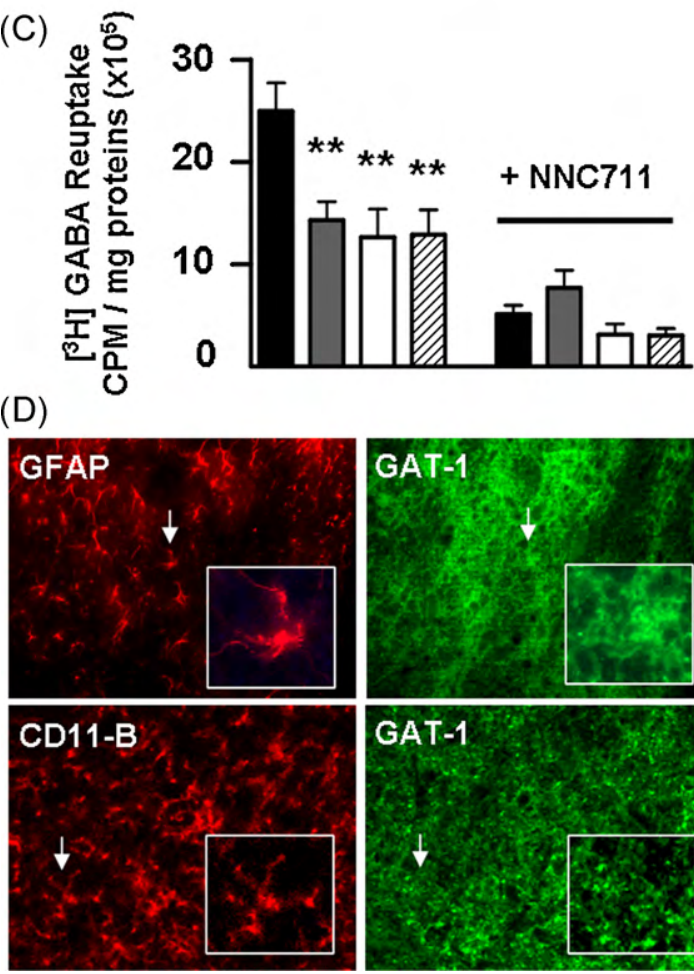

SNI ipsi
$\square$ SNI cont

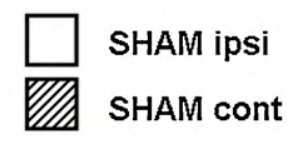

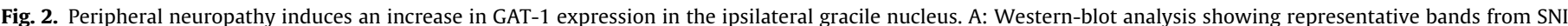

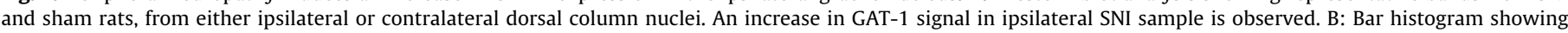

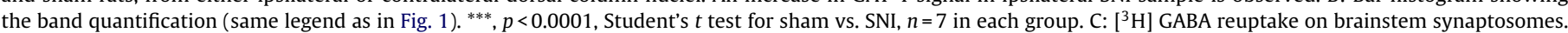

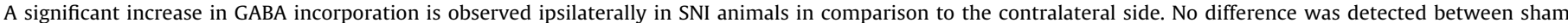

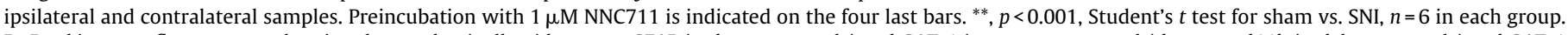

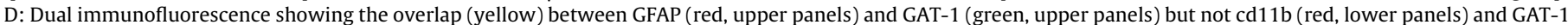
(green, lower panels) signals. Inserts show higher magnification of a representative GFAP labeled astrocytes. Scale bar: $100 \mu \mathrm{m}$.

group, Fig. 2C). Preincubation of synaptosomes with $1 \mu \mathrm{M}$ NNC711 (a GAT blocker that selectively blocks GAT-1 at this concentration) inhibits both ipsi- and contralateral transport up to similar levels $(510,600 \pm 87,000$ vs. 7,683,000 $\pm 173,800)$, suggesting that the increased ipsilateral GABA reuptake in SNI animals was due to
GAT-1 upregulation. Importantly, dual fluorescence immunolabeling reveal that GAT- 1 colocalizes with GFAP signal but not with the microglial marker cd11b (Fig. 2D).

Furthermore, we asked whether the astroglial reaction and the GAT-1 increase were present uniformly throughout the rostro- 

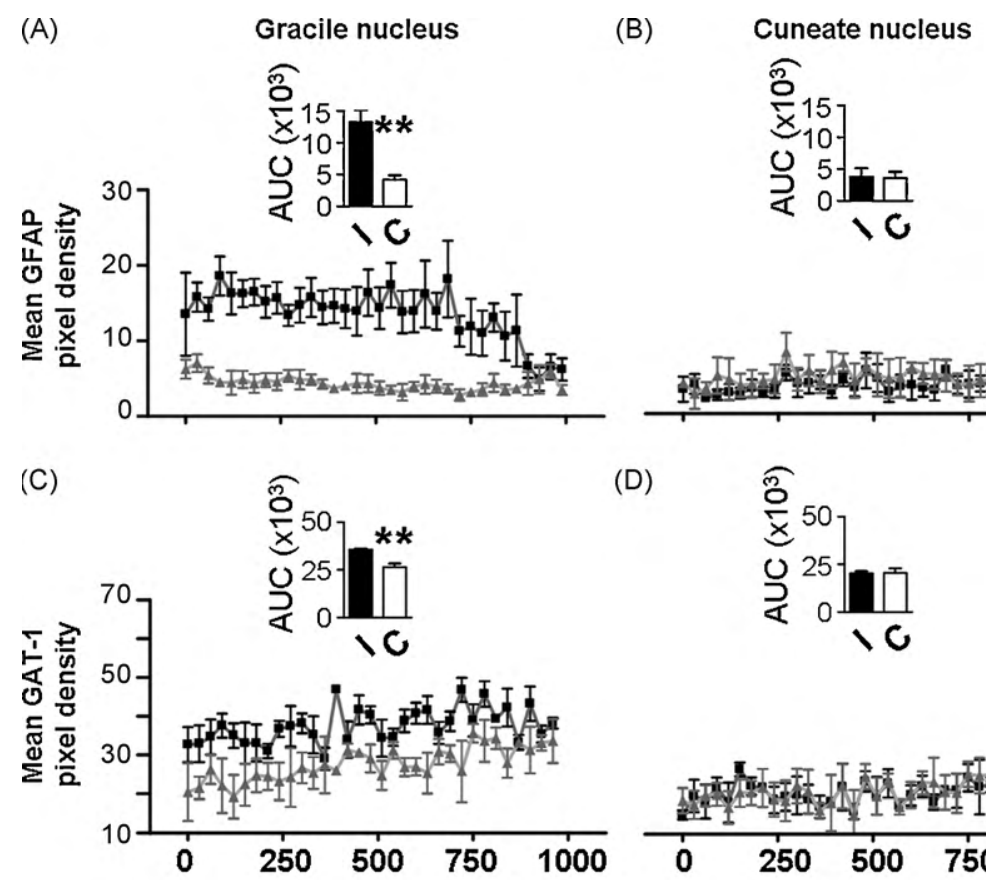

(D)
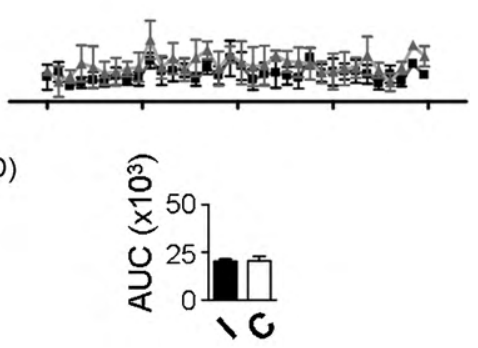

$\mu \mathrm{m}$ from gracile caudal end

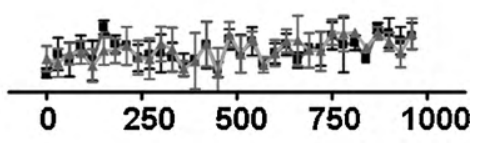

$\mu \mathrm{m}$ from cuneate caudal end

(E) SNI IPSILATERAL SNI CONTRALATERAL
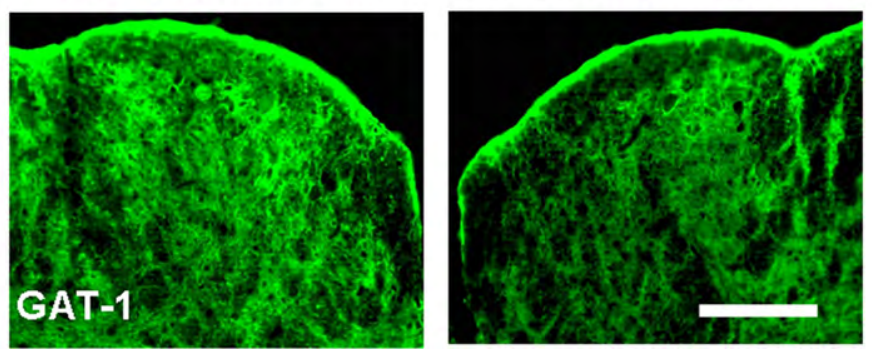

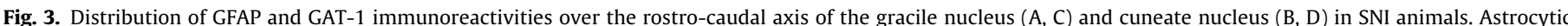

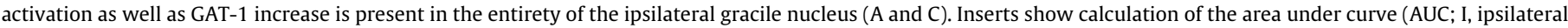

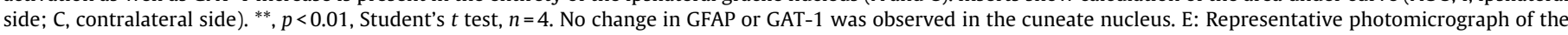
augmented GAT-1 immunoreactivity in the ipsilateral gracile nucleus. Scale bar: $200 \mu \mathrm{m}$.

caudal axis of the gracile nucleus. Mapping analysis shows that the augmentation in both GFAP and GAT-1 immunoreactivity are detectable all along the nucleus (Fig. 3). Interestingly, no change in GFAP or GAT-1 immunosignals could be detected in the caudate nucleus, in line with the targeted glial reaction in regions receiving inputs from the lower limb. As shown in Fig. 3E, this increase is also highlighted using immunofluorescence.

In the present study we report that a glial reaction takes place in the gracile nucleus in the SNI model of neuropathic pain, and show that it is associated with an increase in GAT- 1 expression and an augmented GABA transport capacity. The GAT-1 upregulation was observed throughout the entire gracile nucleus, and was not accompanied with detectable changes in the neighboring caudate nucleus.

These advances are in accordance with previously published works showing that animal models of neuropathy were accompanied with a glial reaction in the gracile nucleus $[6,8,19]$. However, we report here the first study aiming at evaluating the consequences of peripheral nerve injury on the expression of transporters for neurotransmitters in the gracile nucleus. The increase in GAT-1 reported herein is of importance considering the key role played by the GABA reuptake machinery in the maintenance of normal neurotransmission, especially in the somatosensory pathway [5]. Indeed, an increased expression in GAT-1 was reported in the spinal cord of neuropathic pain models, and the associated pain hypersensitivity was reversible upon GAT-1 inhibitor administration [2]. Moreover, engineered mice devoid of the GAT-1 gene show a significant hypoalgesia [23]. It is therefore likely that the GAT1 upregulation contributes to an augmented GABA clearance and favors disinhibition and at least partially results in a perturbation in the normally non-nociceptive signals converging to the gracile nucleus, and then in turn may account for the allodynic behaviors. Furthermore, as it has been previously highlighted that the dorsal column pathway conveys allodynic information as well [14], the observed changes might also account for the increased response to normally painful stimuli (hyperalgesia). Pharmacological antago- 
nism of GAT-1 functioning at the gracile level in SNI rats, as well as GAT- 1 activation in naive animals would clarify this point in the future.

Interestingly, no changes in any analyzed glutamate transporters were found in the present study. This could represent a striking difference from the spinal cord glial regulation in neuropathic pain as a drop in EAAT expression has been recurrently reported in the spinal cord in various models of chronic pain including neuropathy $[1,15,17,20]$. It is conceivable that the signals triggering the glial activation and transporters regulation differ between the spinal cord and the dorsal column pathway. Such signaling remains to be elucidated. Nevertheless, we cannot rule out the possibility that EAAT regulation might take place at another time point during the course of neuropathy.

The possibility that glial perturbation may take place in the dorsal column nuclei in chronic pain offers new perspective for future therapeutics aiming at selectively reducing allodynia or paresthesia/dysesthesia. Interestingly, the dorsal column pathway has emerged as a target in refractory forms of chronic pain through the development of spinal stimulation [7]. Such electrical stimulation has been shown to provide pain relief, although its precise mechanisms of action remain unknown. One possibility would be the interference with the normal physiology of the dorsal column pathway. It would be of primary importance to assess the effect of such stimulation on the glial phenotype in the gracile nucleus. Furthermore, as in the specific context of visceral hyperalgesia the dorsal column pathway has been shown to play a key role [13,21,22], further investigations studying the gracile glial reaction in models of visceral pain would be highly significant.

In conclusion, the present study provides direct evidence of a strong glial reaction together with a marked increase in the GABA transporter GAT-1 in the gracile nucleus in response to peripheral nerve injury. This may have important consequences in our understanding of neuropathy-associated allodynia and paresthesia, and highlight the GABA-mediated inhibition in the dorsal column nuclei as a possible future target to alleviate these symptoms.

\section{Acknowledgements}

This work was supported by the Swiss National Science Foundation (ID) and the International Association for the Study of Pain (RDG). The authors thank Marie Pertin and Guylène Magnin for their technical assistance in the SNI surgery. We are grateful for the support provided by Pr. Christian Kern, from the Department of Anesthesiology, University Hospital Center, Lausanne.

\section{References}

[1] B.C. Binns, Y. Huang, V.M. Goettl, K.V. Hackshaw, R.L. Stephens Jr., Glutamate uptake is attenuated in spinal deep dorsal and ventral horn in the rat spinal nerve ligation model, Brain Res. 1041 (2005) 38-47.
[2] M.A. Daemen, G. Hoogland, J.M. Cijntje, G.H. Spincemaille, Upregulation of the GABA-transporter GAT-1 in the spinal cord contributes to pain behaviour in experimental neuropathy, Neurosci. Lett. 444 (2008) 112-115.

[3] I. Decosterd, C.J. Woolf, Spared nerve injury: an animal model of persistent peripheral neuropathic pain, Pain 87 (2000) 149-158.

[4] S. Echeverry, X.Q. Shi, J. Zhang, Characterization of cell proliferation in rat spinal cord following peripheral nerve injury and the relationship with neuropathic pain, Pain 135 (2008) 37-47.

[5] S.J. Enna, K.E. McCarson, The role of GABA in the mediation and perception of pain, Adv. Pharmacol. 54 (2006) 1-27.

[6] N.P. Eriksson, J.K. Persson, H. Aldskogius, M. Svensson, A quantitative analysis of the glial cell reaction in primary sensory termination areas following sciatic nerve injury and treatment with nerve growth factor in the adult rat, Exp. Brain Res. 114 (1997) 393-404.

[7] S. Falowski, A. Celii, A. Sharan, Spinal cord stimulation: an update, Neurotherapeutics 5 (2008) 86-99.

[8] W. Ma, R. Quirion, Partial sciatic nerve ligation induces increase in the phosphorylation of extracellular signal-regulated kinase (ERK) and c-Jun N-terminal kinase (JNK) in astrocytes in the lumbar spinal dorsal horn and the gracile nucleus, Pain 99 (2002) 175-184.

[9] S.B. McMahon, M. Malcangio, Current challenges in glia-pain biology, Neuron 64 (2009) 46-54.

[10] K. Miki, K. Iwata, Y. Tsuboi, T. Morimoto, E. Kondo, Y. Dai, K. Ren, K. Noguchi, Dorsal column-thalamic pathway is involved in thalamic hyperexcitability following peripheral nerve injury: a lesion study in rats with experimental mononeuropathy, Pain 85 (2000) 263-271.

[11] K. Miki, K. Iwata, Y. Tsuboi, R. Sumino, T. Fukuoka, T. Tachibana, A. Tokunaga, K. Noguchi, Responses of dorsal column nuclei neurons in rats with experimental mononeuropathy, Pain 76 (1998) 407-415.

[12] E.D. Milligan, L.R. Watkins, Pathological and protective roles of glia in chronic pain, Nat. Rev. 10 (2009) 23-36.

[13] J. Palecek, The role of dorsal columns pathway in visceral pain, Physiol. Res. 53 (Suppl. 1) (2004) S125-S130.

[14] H. Sun, K. Ren, C.M. Zhong, M.H. Ossipov, T.P. Malan, J. Lai, F. Porreca, Nerve injury-induced tactile allodynia is mediated via ascending spinal dorsal column projections, Pain 90 (2001) 105-111.

[15] B. Sung, G. Lim, J. Mao, Altered expression and uptake activity of spinal glutamate transporters after nerve injury contribute to the pathogenesis of neuropathic pain in rats, J. Neurosci. 23 (2003) 2899-2910.

[16] M.R. Suter, T. Berta, Y.J. Gao, I. Decosterd, R.R. Ji, Large A-fiber activity is required for microglial proliferation and p38 MAPK activation in the spinal cord: different effects of resiniferatoxin and bupivacaine on spinal microglial changes after spared nerve injury, Mol. Pain 5 (2009) 53.

[17] Y.H. Tai, Y.H. Wang, J.J. Wang, P.L. Tao, C.S. Tung, C.S. Wong, Amitriptyline suppresses neuroinflammation and up-regulates glutamate transporters in morphine-tolerant rats, Pain 124 (2006) 77-86.

[18] Y.X. Tao, J. Gu, R.L. Stephens Jr., Role of spinal cord glutamate transporter during normal sensory transmission and pathological pain states, Mol. Pain 1 (2005) 30.

[19] R. Terayama, S. Omura, N. Fujisawa, T. Yamaai, H. Ichikawa, T. Sugimoto, Activation of microglia and p38 mitogen-activated protein kinase in the dorsal column nucleus contributes to tactile allodynia following peripheral nerve injury, Neuroscience 153 (2008) 1245-1255.

[20] H.R. Weng, N. Aravindan, J.P. Cata, J.H. Chen, A.D. Shaw, P.M. Dougherty, Spinal glial glutamate transporters downregulate in rats with taxol-induced hyperalgesia, Neurosci. Lett. 386 (2005) 18-22.

[21] W.D. Willis, E.D. Al-Chaer, M.J. Quast, K.N. Westlund, A visceral pain pathway in the dorsal column of the spinal cord, Proc. Natl. Acad. Sci. U.S.A. 96 (1999) 7675-7679.

[22] W.D. Willis Jr., K.N. Westlund, The role of the dorsal column pathway in visceral nociception, Curr. Pain Headache Rep. 5 (2001) 20-26.

[23] Y.F. Xu, Y.Q. Cai, G.Q. Cai, J. Jiang, Z.J. Sheng, Z.G. Wang, J. Fei, Hypoalgesia in mice lacking GABA transporter subtype 1, J. Neurosci. Res. 86 (2008) 465-470. 